Dovale Carrión, Carmiña.

Profesora Asociada, Escuela Politécnica de Donostia de la Universidad del País Vasco, Departamento de Expresión Gráfica y Proyectos de Ingeniería.

\title{
Gravedad y levitación en la obra de Eduardo Chillida.
}

TIPO DE TRABAJO

Comunicación

PALABRAS CLAVE

Chillida, gravedad, levitación

KEY WORDS

Chillida, gravity, levitation

\section{RESUMEN}

El hecho de ser escultura implica para Eduardo Chillida hablar de peso, de gravedad. Si algo está físicamente en el espacio es precisamente porque pesa, porque las leyes de la gravedad le afectan. Incluso los objetos-idea dibujados a lápiz van a sufrir los efectos de la gravedad y buscarán su estabilidad a través de otros elementos de la composición.

Pero hablar de gravedad no significa poner una gran cantidad de materia en contacto con el suelo. Todo lo contrario. El autor intenta evadirse de su efecto de forma que su presencia se haga todavía más fuerte. Para ello, utilizará la fuerza de la levitación, una fuerza que actúa justo en sentido contrario a la de la gravitación, y que vincula el elemento con el cielo en lugar de con la tierra. Gravitación vs levitación no deja de ser el binomio cuerpo y alma.

El objetivo final de esa lucha entre gravitación y levitación es sin duda que el elemento quede en equilibrio. Pero ese equilibrio no se referirá únicamente a un equilibrio estático de fuerzas, sino también a un equilibrio formal basado en la búsqueda de los extremos que llevará a una mayor expresividad de la obra.

\section{ABSTRACT}

Eduardo Chillida thinks that it's absolutely necessary to talk about weight and gravity when we refer to sculpture. If something is in the space, it's because of its weight, because it's affected by the gravitation law. Even the objects-idea of Chillida drawn by pencil will suffer the gravitation effects, and will look for their stability using other elements of the composition.

But to talk about gravitation is not to put a big amount of material in direct contact to the ground. It's just the opposite. The author tries to avoid the effect of gravitation to do its presence even stronger. To do that, he will use the force of levitation, a force that acts in the opposite sense of gravitation, and links the element to the sky and not to the ground. Gravitation vs levitation is like body vs soul.

The final objective of this fight between gravitation and levitation is to reach the balance. But this balance won't refer only to a balance of forces, but also to a form balance based in the search of extremes that will lead to a bigger expressivity of the work. 


\section{CONTENIDO}

"Yo me rebelo contra Newton. Yo y mis esculturas"”, afirma Chillida.

La horizontal y la vertical aparecen constantemente en la obra de Eduardo Chillida. El eje " $x$ " marca el horizonte y la estabilidad, mientras que el eje " $y$ " habla de la relación entre cielo y tierra, entre gravitación y levitación.

La verticalidad es símbolo de vida. El ser humano la gana poco a poco, y la pierde en el momento de su muerte. Por tanto, estar de pie implica un gran esfuerzo, pero es también una posibilidad de vida. "Hombre encendido/Árbol/Hombre apagado/Agua/Recostada invertida/Quieto lago" ${ }^{2 \prime}$.

Por el simple hecho de que nuestro cuerpo pesa, estamos ligados de manera directa a la tierra. Y aunque intentemos escapar de su efecto, sabemos que volveremos sobre ella una y otra vez. Pero esto únicamente se refiere a la parte física del humano; la parte espiritual es mucho más libre, y se asocia con frecuencia al cielo, precisamente por su inmaterialidad. La lucha entre gravitación y levitación no deja de ser una lucha entre cuerpo y alma. Chillida habla de una batalla irracional contra la gravedad, de una "gran batalla que ocurre en la vertical/entre las fuerzas que suben y las fuerzas que bajan/la misma batalla que existe en las líneas curvas/entre lo centrípeto y lo centrífugo,/entre la convexidad y la concavidad ${ }^{3 "}$.

Las obras de Eduardo Chillida pesan toneladas, y ello exige un contacto directo con el suelo para poder asegurar su descarga. Podríamos decir incluso que parece que nacen del suelo. Pero como explica Werner Schmalenbach, "si bien todo el arte de Chillida tiene la condición del peso, también le fascina el vencerlo, pero no por la ligereza sino por la ingravidez a través de la cual se experimenta el peso ${ }^{4 \prime}$. Es entonces cuando las obras arraigadas a la tierra se abren al cielo como un árbol. Y ese diálogo es tan intenso, que a veces parece que dejan de pesar para comenzar a volar.

A continuación, varios ejemplos de obra en los que el eje vertical juega un papel protagonista servirán para analizar el vínculo entre cielo y tierra.

\section{1- Apertura de la forma.}

En el caso de "Buscando la luz I" (1997), tres planchas de acero definen un recinto en forma de "u" en donde el espectador puede entrar. La apertura de la forma en distintas etapas a través de geometrías curvas indica la dificultad de subir en altura, y crea el vínculo con el cielo. El visitante, una vez dentro de la obra y protegido por las planchas, comprende cómo va creciendo el espacio interior con la altura. Parece incluso que el material es cada vez más ligero. Y tras un rato de observación, abandonamos el suelo para viajar mentalmente hacer arriba, hacia la luz y el cielo.

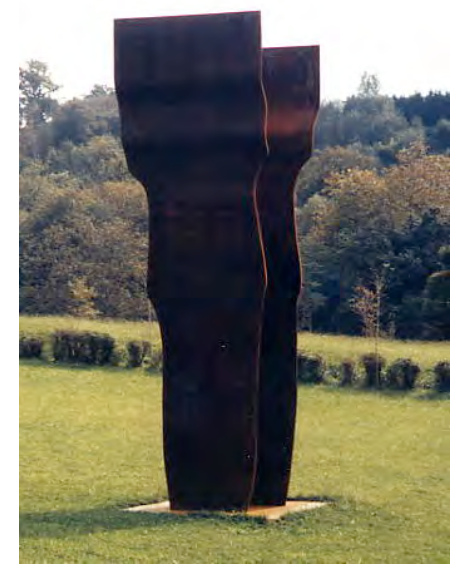

Ilustración 1. Buscando la luz I (1997007)

\footnotetext{
${ }^{1}$ Chillida Belzunce, Susana. Elogio del horizonte. Ediciones Destino, Barcelona, 2003. Pág. 16.

${ }^{2}$ Chillida, Eduardo. Escritos. La Fábrica, Madrid, 2005. Pág. 36.

${ }^{3}$ Chillida, Eduardo. Chillida, doctor honoris causa. Escuela Técnica Superior de Ingenieros Industriales y de Ingenieros de Telecomunicación, Bilbao, 1998. Pág. 15.

${ }^{4}$ Barañano, Kosme María de, José Ângel Valente, Werner Schmalenbach, y Gabriel Celaya. Chillida en San Sebastián. Sociedad

Guipuzcoana de Ediciones y Publicaciones, Donostia-San Sebastián, 1992. Pág. 60.
} 
El peso de la escultura descarga en este caso a través de las tres líneas de intersección de las planchas con el suelo. Pero la apertura de la forma define un eje de tensión hacia arriba. Entre los dos, el escultor define un instante de equilibrio.

2- Unión física entre la materia y el vacío para la definición de la vertical.

El hecho de recortar una parte del material posibilita la entrada de aire en el interior de la obra "Elogio al cubo_Homenaje a Juan de Herrera". La geometría de ese vacío se compone de dos partes; una primera parte rectangular vertical que acaba en una segunda de geometría cuadrada. Esta entrada de aire tiene un gran parecido formal con el acceso a una cueva, en donde normalmente hay un espacio estrecho de acceso conectado con un espacio central de mayor dimensión. El cuadrado de vacío, protagonista de la obra, no podrá salir de nuevo al exterior ya que no hay sitio. Está atrapado, y ésa es la estrategia para asegurar la unión entre materia y vacío.

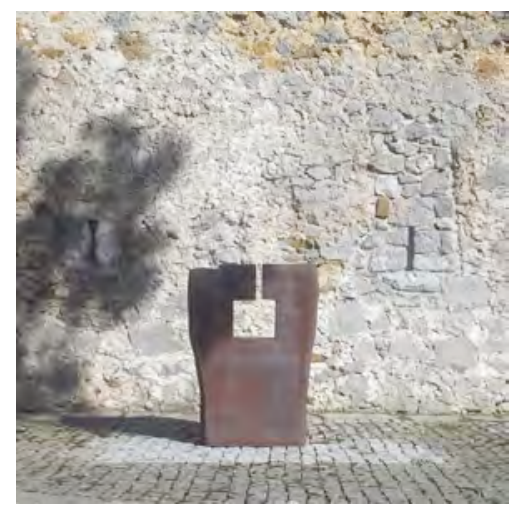

\section{Ilustración 2. Elogio al cubo_Homenaje a Juan de Herrera (1990005)}

La obra apoya en el suelo, pero la presencia de una unión con el vacío en su parte superior genera una tensión que la lleva hacia arriba. ¿Quién soporta entonces el peso de la escultura? ¿El cielo o la tierra?

3- Protagonismo de la tensión horizontal frente a la vertical como estrategia para levitar.

El « Homenaje a Luca Pacioli » es una gran mesa rectangular de acero (con uno de sus lados curvo), que descansa en el suelo a través de tres pequeños apoyos colocados en su parte inferior, y dispuestos 2 a 1 . El hecho de no poder ver los tres apoyos a la vez, dado que el punto de vista del espectador es mucho más alto, y que la propia geometría de la obra los esconde, genera en el espectador la ilusión de la levitación. Da entonces la impresión de que "es el aire el que mantiene la pieza suspendida en el suelo". Una pieza que, además, parece avanzar hacia su parte curva.

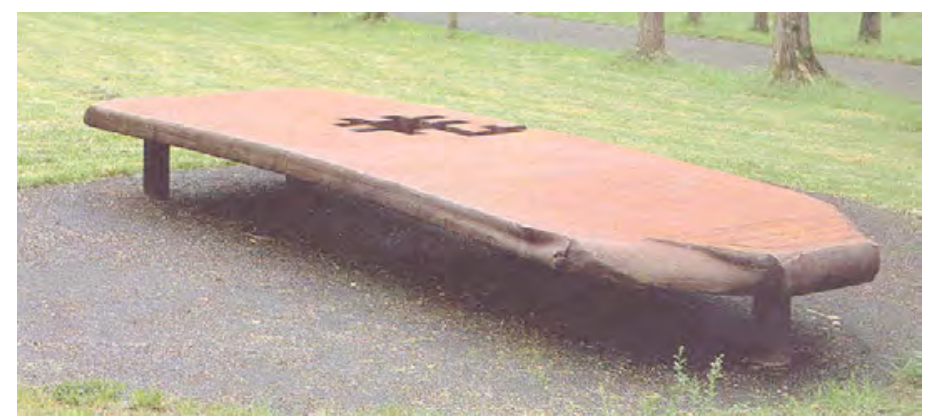

\section{Ilustración 3. Homenaje a Luca Pacioli (1986003)}

En este caso, el autor da mucho más protagonismo al plano horizontal que al vertical. De hecho, él mismo explica que "el objetivo de las mesas es ver hasta dónde puede uno arrimarse a las dos dimensiones y conseguir que la obra siga siendo una escultura ". Eso hace que la tensión vertical se pierda, y que parezca que únicamente se trabaja en un plano horizontal distinto al del suelo, lo que sugiere que la mesa flota.

\footnotetext{
${ }_{6}^{5}$ Martínez Aguinagalde, Florencio. Palabra de Chillida. Servicio Editorial de la Universidad del País Vasco, Bilbao, 1998. Pág. 41.

${ }^{6}$ Ibíd.
} 


\section{4- Penetrar el aire para elevarse.}

El propio título de esta obra hace pensar en el cielo: "El vuelo de los pájaros" (1952). El contacto con el suelo se produce a través de una piedra de la cual emerge un elemento metálico vertical central. De ahí, surgen nuevos elementos que penetran el aire en todas sus direcciones, como verdaderos pájaros. La relación con el espacio es tan intensa, que acabamos por olvidar el vínculo con el suelo. Y eso no deja de ser una forma de elevarse.

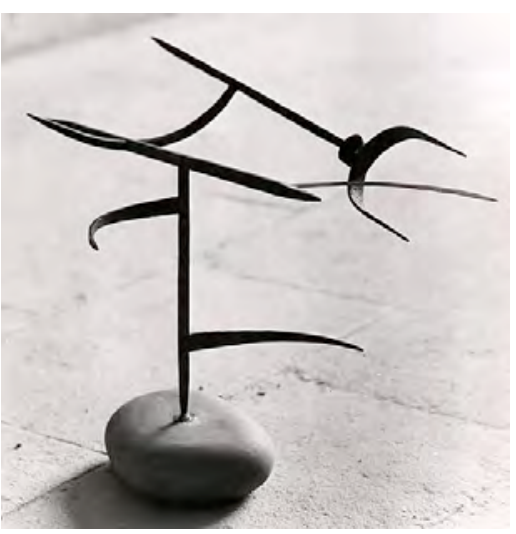

Ilustración 4. El vuelo de los pájaros (1952004)

En este caso, el hecho de que los elementos protagonistas de la obra hagan referencia directa a elementos del cielo hace olvidar la fuerza de la gravitación.

5- Relación con el cielo como una forma de localizar al espectador para que pueda mirar al horizonte.

La escultura "Elogio al Horizonte IV" situada en el Cerro de Santa Catalina de Gijón abraza el aire con un gesto que invita a los visitantes a entrar. Una vez dentro, se establece una conexión vertical con el cielo a través del dibujo de una elipse en la parte superior de la obra. Ese eje vertical establece la posición exacta desde la cual el espectador observará el horizonte a través de una ventana recortada en el muro de hormigón.

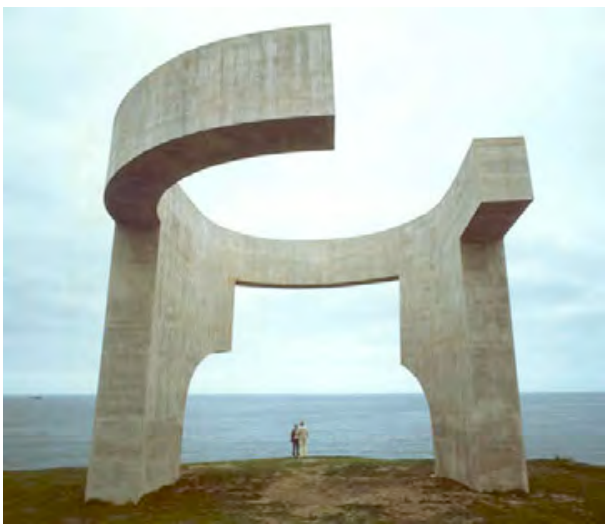

Ilustración 5. Elogio al Horizonte IV (1989070)

Esta obra pretende por tanto materializar una posición en el espacio e indicar una dirección clara hacia la que el espectador debe mirar. Podría haber planteado únicamente un recinto cerrado con una ventana abierta al mar, pero el efecto es mucho más potente si se establece un vínculo con el cielo antes de mirar al horizonte. Es como si se estirase al visitante hacia arriba antes de hacerle caminar mentalmente hacia delante, en un estado de apertura pura.

Eduardo Chillida no sólo estudia el tema de la gravitación y levitación mediante sus esculturas, sino también mediante su obra gráfica. Entiende el conjunto de su obra como escultura, y, por tanto, trabaja los mismos temas independientemente del soporte que utilice. Inventa una técnica denominada "gravitación" que consiste en disponer varios papeles cosidos entre sí, y colgar el conjunto de la pared a través de un hilo y un par de chinchetas. El hecho de coser crea puntos de unión directa entre las superficies, pero las deja libres el resto del tiempo. El observador identificará entonces distintas capas y el aire existente entre ellas, lo que generará una fuerte 
tridimensionalidad. Según Kosme de Barañano, en las gravitaciones "Chillida juega con el concepto de gravitar casi en el límite, pues el papel no pesa. Al suspenderse, los papeles se enclavan, enmarcan o desmarcan entre ellos, es decir, se articulan en relieve. La definición, esto es, la limitación de los planos señalados o concretados por el papel o su mancha, ahora vacila, cuelga y levita desde los puntos-línea que los mantienen". Veamos el tema de la gravitación y levitación en este contexto a través del ejemplo "Gravitación Tinta 1988034".

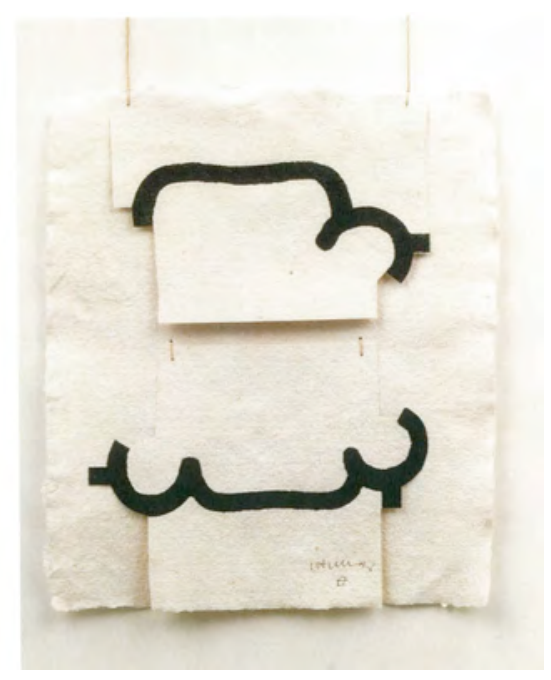

\section{Ilustración 6. Gravitación Tinta 1988034}

Esta gravitación se compone de tres papeles. Hay un papel de base de gran dimensión que cuelga de la pared a través de un hilo y dos chinchetas, que están perfectamente alineadas con los puntos de perforación de la base. Sobre ella, se cosen dos nuevos papeles de menor dimensión, uno sobre el otro. Y sobre cada uno de estos papeles secundarios, el autor dibuja una línea de tinta. El diálogo entre las dos líneas será el elemento compositivo principal de la obra.

Los dos papeles secundarios no son únicamente un soporte físico sobre el cual el autor dibuja, sino que forman parte de la propia creación de la línea. Los límites del papel acompañan a la tinta en su dibujo, y sirven para generar unos límites más difusos. En el detalle siguiente, se ve cómo la geometría del papel sigue dibujando el arco ya definido por la tinta.

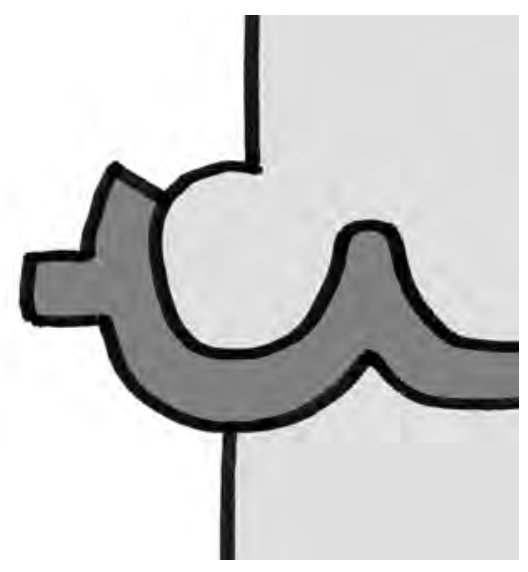

Ilustración 7. Esquema de la creación de un arco en la "Gravitación Tinta 1988034"

La línea curva es una manera muy sencilla de atrapar el espacio. El hecho de curvarse hace que la línea no atraviese el espacio, sino que lo envuelva. Cada una de las líneas de tinta de la composición se compone de arcos "puros" y de un tipo de línea compuesta por una parte central horizontal y dos pequeños arcos de cerramiento en sus extremos. Podríamos decir que se trata de un arco más

\footnotetext{
${ }^{7}$ Barañano, Kosme María de, Ina Busch, Matthias Bärmann, Tomás Llorens, y José Ángel Valente. Chillida 1948-1998. Museo Nacional Reina Sofía, y Aldeasa, Madrid, 1998. Pág. 45.
} 
"lento". La disposición de un arco "lento" central acompañado de dos arcos "puros" laterales constituye la estructura formal principal de esta obra.

Este esquema formal no se repite exactamente de la misma manera en las dos líneas. La línea superior, no1, no tiene un arco "puro" a la izquierda, y esa ausencia juega un papel protagonista. Por un lado, se evita una simetría demasiado rígida; y por otro, el hecho de que la línea superior encadene dos arcos, y la inferior tres, establece un diálogo entre lo binario y lo ternario. El número tres aparece en la parte inferior, lo que hace pensar en un mejor apoyo del peso de la obra.

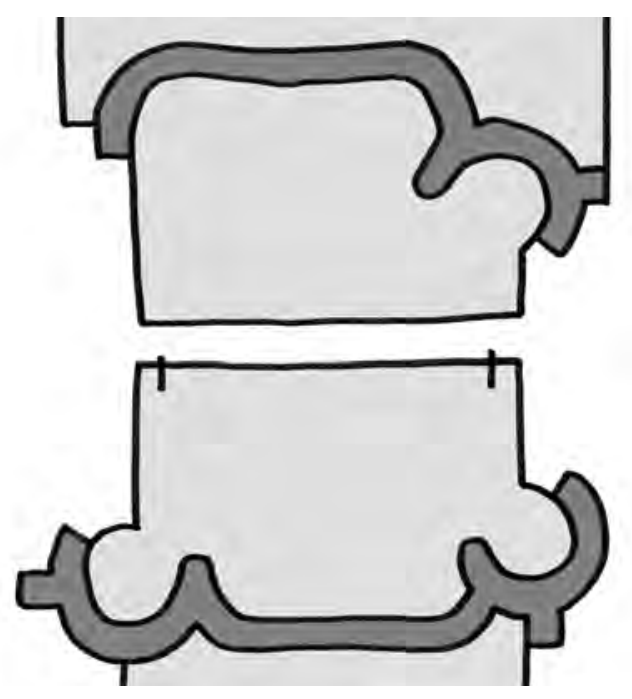

Ilustración 8. Esquema de las dos líneas de tinta de la "Gravitación Tinta 1988034"

Los límites de los papeles secundarios, al participar directamente en la elaboración de las geometrías, ofrecen unos cambios de forma justificados que generan movimiento en la composición. Por ejemplo, la anchura del papel secundario no1 (superior) se estrecha después de la definición de la línea de tinta. El papel nำ2 sin embargo, nace estrecho, con la misma anchura que el final del papel no1, y se ensancha para acoger a la segunda línea de tinta. Una vez que la línea ya se ha definido, se vuelve de nuevo más estrecho para no dar mucho peso a la composición, visto que es justo la parte inferior de la obra. Se podría incluso llegar a pensar que el papel no1 entre en la base para salir de nuevo convertido en $n \mathbf{2} 2$, como si se cosiera todo el papel secundario a la base. Al observar la obra en general, aparece una nueva relación. La anchura del papel no1 en su parte superior, coincide con la anchura de la línea de tinta no2, lo que cierra el círculo y otorga coherencia a la obra.

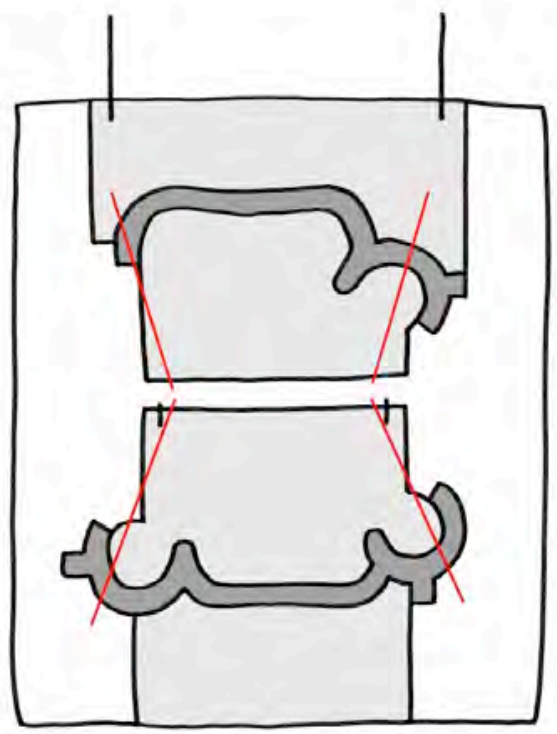

Ilustración 9. Esquema de cambio de forma en el eje vertical de la “Gravitación Tinta 1988034" 
Pero el movimiento de la obra no se produce únicamente en el eje vertical "y". El ojo del lector viaja de arco "puro" en arco "puro", debido a su semejanza formal, generando un movimiento cíclico en el interior de la composición. El hecho de que los dos arcos de la línea inferior estén a distinta altura, por ejemplo, ayuda en la génesis del movimiento. Y los pequeños segmento de tinta que emergen de los arcos, todos con distinta dirección y sentido, son la respuesta a ese movimiento interior, ya que intentan marcar el cambio de dirección. De esta forma, se procura llegar al equilibrio de la composición.

La línea $\mathrm{n}$-1 de geometría convexa construye un techo, y, sin embargo, la línea n2, un suelo. Dicho de otro modo, la línea no1 abraza el espacio que queda bajo ella, y la línea no2, el que hay sobre ella. Finalmente, en la distancia, están intentando aprehender un mismo espacio intersticial central. El resultado de esta obra es, por tanto, la definición de un espacio "entre" líneas.

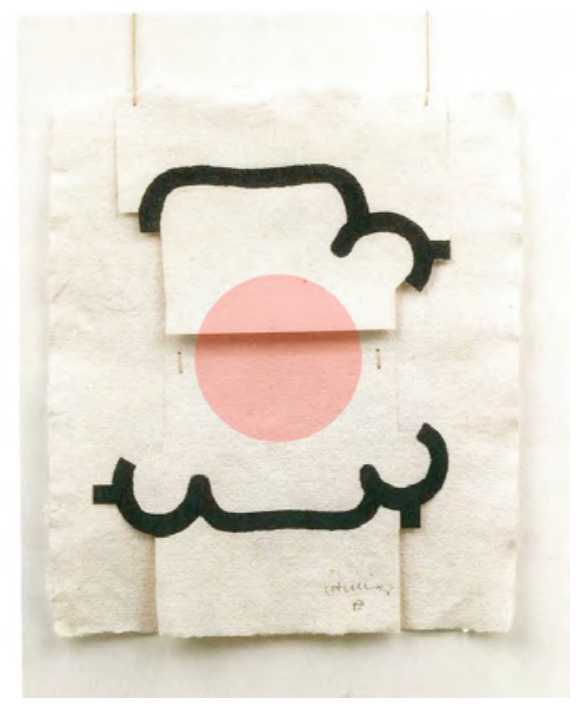

\section{Ilustración 10. Esquema del espacio “entre" de la "Gravitación Tinta 1988034"}

Volviendo al tema que nos ocupa, la línea no1 está vinculada a la elevación. Parece querer subir hacia arriba, y sigue con ese gesto la tensión de los hilos que sostienen su papel secundario. La línea no2, sin embargo, habla de gravitación, y parece querer desplazarse hacia abajo, en sentido opuesto a la tensión de los hilos que sostienen su papel secundario. La lucha de tensiones en la parte inferior agranda el espacio central y da fuerza a la obra.

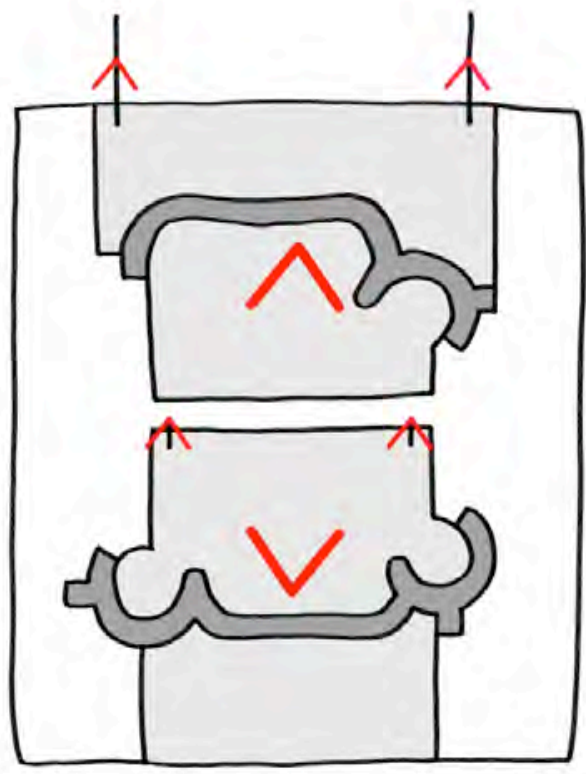

Ilustración 11. Pro-esfuerzo vs contra-esfuerzo en la “Gravitación Tinta 1988034" 
Estas dos líneas que representan la levitación $(\uparrow)$ y la gravitación $(\downarrow)$, y por tanto el cielo y la tierra, aparecen en el contexto de una gravitación, una obra compuesta de varios papeles que en realidad cuelga de un muro a través de un hilo. Un hilo que luchará contra el peso de la tinta para mantener el equilibrio.

Se trata por tanto de un juego de fuerzas en el que la tinta pesa por su forma, en el que el hilo parece desaparecer y ayuda a sugerir la levitación del papel, y en el que no se sabe si las líneas de tinta quieren definir un espacio "entre" o es ese espacio "entre" el que hace que las líneas no se vayan.

La obra de Eduardo Chillida no levita, pero quiere generar esa ilusión: “A tu,/artífex de la ingravidesa/del massís".

Para conseguirlo, utilizará la estrategia de la forma. Serán geometrías que señalarán el cielo. Las formas generarán espacios más grandes a medida que se sube en altura, introducirán uniones materia-aire, penetrarán el espacio en todas sus direcciones, etc. La técnica de la gravitación le permitirá hablar del peso de la tinta y del papel, cuando en realidad el conjunto se descuelga de la pared, como si proviniera del cielo.

Y mediante esa activación del eje vertical, la obra mirará hacia arriba intentando alejarse del contacto directo con la tierra. Gravitación y levitación quedarán entonces compensadas, y la obra adquirirá mayor intensidad. Parecerá más ligera, querrá moverse y cobrará vida.

“Gravitación y levitación es la vertical. La vertical es la gran línea del hombre. La vertical del hombre vivo, ¿verdad? Es decir, está formada, está condicionada por la gravedad, por la gravitación, pero en la misma línea actúa otra fuerza que se rebela contra la gravedad de Newton y que es una fuerza que va hacia arriba, es..., pueden ser muchos grados de espiritualidad, puede llegar hasta los místicos, las utopías, todo..., la religión, todo actúa en esa línea, y ésa es mi línea"”."

\section{FUENTES REFERENCIALES.}

bARAÑANO, Kosme María de, Ina BUSCH, Matthias BäRMANN, Tomás LLORENS, y José Ángel VALENTE. Chillida 1948-1998. Madrid: Museo Nacional Reina Sofía, y Aldeasa, 1998. 374 p. ISBN: 84-8026-120-X

BARAÑANO, Kosme María de, José Ángel VALENTE, Werner SCHMALENBACH, y Gabriel CELAYA. Chillida en San Sebastián. DonostiaSan Sebastián: Sociedad Guipuzcoana de Ediciones y Publicaciones, 1992. 400 p. ISBN: 8471731967

BEAUMONT, Marutxi. Arquitectura de l'aire. Cerdanyola del Vallès: Editorial Montflorit, 2008. 64 p. ISBN: 978-95705-70-9

BROSSA, Joan, y Eduardo CHILLIDA. Brossa i Chillida a peu per llibre. Barcelona: Polígrafa, 1996. 76 p. ISBN: 978-84-343-0809-1

CHILLIDA, Eduardo. Chillida, doctor honoris causa. Bilbao: Escuela Técnica Superior de Ingenieros Industriales y de Ingenieros de Telecomunicación, 1998. 150 p. ISBN: 8483734656

CHILLIDA, Eduardo. Escritos. Madrid: La Fábrica, 2005. 124 p. ISBN: 84-96466-03-5

CHILLIDA BELZUNCE, Susana. Elogio del horizonte. Barcelona: Ediciones Destino, 2003. 210 p. ISBN: 84-233-3552-6

MARTínez AguinagALDE, Florencio. Palabra de Chillida. Bilbao: Servicio Editorial de la Universidad del País Vasco, 1998. 120 p. ISBN: 84-7585-950-X

UGARTE, Luxio. Chillida: dudas y preguntas. Donostia-San Sebastián: Erein Argitaletxea, 1995. 144 p. ISBN: 84-7568-574-9

\footnotetext{
${ }^{8}$ "A ti/artifice de la ingravidez/del peso"

Beaumont, Marutxi. Arquitectura de l'aire. Editorial Montflorit, Cerdanyola del Vallès, 2008. Pág. 57.

${ }_{9}^{9}$ Ugarte, Luxio. Chillida: dudas y preguntas. Erein Argitaletxea, Donostia-San Sebastián, 1995. Pág. 55, 56.
} 